\title{
Gemeinsame Verheißung und gemeiner Nutzen
}

\author{
Ein Beitrag für eine aktuelle Würdigung der Confessio \\ Augustana als gemeinsames ökumenisches Dokument
}

\section{Einleitung}

Wenn man das Augsburger Bekenntnis heute als mögliches gemeinsames ökumenisches Dokument betrachtet, stellt sich die Frage, was sich seit den intensiven Diskussionen in den Jahren vor 1980 geändert hat. ${ }^{1}$ Damals gab es sowohl eine große Menge sehr tiefer Analysen des Bekenntnistextes als auch intensive Diskussionen über eine mögliche gemeinsame Anerkennung von katholischer und lutherischer Seite. ${ }^{2}$ Die damals vorgebrachten Gedanken und Argumente sollen an dieser Stelle nicht wiederholt werden. Die Intention dieses Beitrags ist es vielmehr, zu einem Perspektivwechsel in der Diskussion anzuregen und möglicherweise auch neue Erkenntnisse in das noch laufende Gespräch einzubringen.

Ich werde mich im Folgenden auf drei Punkte beschränken, die m. E. in die Diskussion eingebracht werden müssen: ${ }^{3}$ die neue gesellschaftliche, kirchliche und politische Lage der Gegenwart im Vergleich zur Situation um 1980, des Weiteren die veränderte Haltung der Verfasser der Gemeinsamen Erklärung zur Rechtfertigungslehre von 1999 im Vergleich zum Selbstverständnis der Diskussi-

1 Für die sprachliche Überprüfung dieses Beitrags danke ich Dr. Kinga Zeller und Dr. Axel Lange. 2 S. z.B. Harding Meyer/Heinz Schütte (Hg.), Confessio Augustana. Bekenntnis des einen Glaubens. Gemeinsame Untersuchung lutherischer und katholischer Theologen, Paderborn/Frankfurt a. M. 1980; Peter Gauly, Katholisches Ja zum Augsburger Bekenntnis? Ein Bericht über die neuere Anerkennungsdiskussion, Freiburg i.B. 1980; Harding Meyer/Heinz Schütte/Hans-Joachim Mund (Hg.), Katholische Anerkennung des Augsburgischen Bekenntnisses? Ein Vorstoß zur Einheit zwischen katholischer und lutherischer Kirche, Frankfurt a.M. 1977 (Ökumenische Perspektiven 9); Fritz Hoffmann/Ulrich Kühn (Hg.), Die Confessio Augustana im ökumenischen Gespräch, Berlin 1980; Johannes Brosseder, Die Anerkennung der Katholizität der Confessio Augustana und ihre ekklesiologischen Implikationen. Historische und fundamentaltheologische Probleme, in: Friedrich Mildenberger/Joachim Track (Hg.), Zugang zur Theologie. Fundamentaltheologische Beiträge. Wilfried Joest zum 65. Geburtstag, Göttingen 1979.

3 Andere Perspektiven, wie bspw. die Diskussion um die Frage, wie eine gemeinsame Anerkennung der Confessio Augustana aussehen könnte, werden in diesem Beitrag nicht berücksichtigt.

๑ OpenAccess. (C) 2022 Bo Kristian Holm, publiziert von De Gruyter. (cc) BY-NC-ND Dieses Werk ist lizenziert unter einer Creative Commons Namensnennung - Nicht kommerziell - Keine Bearbeitung 4.0 International Lizenz. https://doi.org/10.1515/9783110683868-022 
onspartner von 1980. Dazwischen setze ich einen Exkurs zu humanistischen Diskursen zur Reformationszeit.

Meine These ist, dass sich diese drei Aspekte fruchtbar verbinden lassen. Der zu entfaltende Gedankengang ist folgender:

1) Das Augsburger Bekenntnis als Ausdruck der Wittenberger Theologie behandelt nicht nur innere kirchliche und theologische Fragestellungen, sondern ist auch als Teil der Wittenberger Antwort auf eine grundlegende epistemologische Krise des Renaissance-Humanismus anzusehen. Ein Skeptizismus wie der des frühen 16. Jahrhunderts ist in veränderter Form auch heute wieder aktuell und bleibt somit auch in der Gegenwart eine theologische Herausforderung.

2) Mitten in einer epistemologischen Krise des späten Renaissance-Humanismus repräsentiert die Confessio Augustana einen spezifisch christlichen Blick auf Schöpfung und weltliche Autorität mit tiefen Wurzeln in sowohl biblischer als auch antiker römischer Sozialphilosophie. Im Zentrum dieses Blickes steht der Begriff der promissio, wie er auch für die Rechtfertigungslehre zentral ist.

3) Wenn wir die Diskussion anlässlich der 450-jährigen Feier des Augsburger Bekenntnisses mit der Gemeinsamen Erklärung vergleichen, wird deutlich, dass der Begriff der promissio, der Verheißung, Ende der 1990er Jahre eine ganz andere Rolle spielte als noch 1980. Das könnte auch für ein heutiges Verständnis der Confessio Augustana entscheidend sein. Es dreht sich hier um eine Veränderung in der Selbstwahrnehmung auf beiden Seiten des Gesprächs. ${ }^{4}$ Die Chancen für eine Wahrnehmung des konfessionell Gemeinsamen in der Confessio Augustana scheinen günstiger geworden zu sein.

Um diese drei Punkte miteinander zu verbinden, werde ich meine Ausführungen mit dem letzten Aspekt beginnen, mit der Analyse des gegenwärtigen Diskurshorizontes. Ich verstehe die Gemeinsame Erklärung von 1999 dabei als eine wichtige Zäsur, weil sich die Grundhaltung beider Gesprächspartner, die für die Einschätzung des konfessionell Gemeinsamen von großer Bedeutung ist, seit dieser Zeit nicht wesentlich verändert hat. In der Gemeinsamen Erklärung finden

\footnotetext{
4 Lutherische konfessionelle Identität ist nicht unbeweglich, sondern eine sich in der Geschichte entwickelnde Identität. So lässt sich die Hervorhebung der promissio in beiden Konfessionen am Ende des 20 Jahrhunderts auch als eine erneute Selbstwahrnehmung auf beiden Seiten des Gesprächs verstehen. Zur konfessionellen Variation in der lutherischen Tradition s. z.B. Irene Dingel, Von der Wittenberger Reformation zum Luthertum. Konfessionelle Transformationen, in: Wolfgang Thönissen/Josef Freitag/Augustinus Sander (Hg.), Luther: Katholizität \& Reform. Wurzeln - Wege - Wirkungen, Leipzig/Paderborn 2016, 239-60.
} 
wir zum ersten Mal die offizielle Lehre der jeweiligen Kirchen „offiziell im Dokument selbst“. ${ }^{5}$

\section{Promissio und Gewissheit des Glaubens in der Gemeinsamen Erklärung zur Rechtfertigungslehre}

Es ist bekannt, dass die vielen Studien zur gemeinsamen Anerkennung der Confessio Augustana aus der Zeit um 1980 folgenlos blieben. Wer diese Diskussion heute weiterführen möchte, muss zuerst nach Änderungen im Selbstverständnis der beteiligten Konfessionen fragen. Zur Klärung dieser Frage bietet sich Die Gemeinsame Erklärung zur Rechtfertigungslehre von 1999 als Grundlage an. ${ }^{6}$

In der Gemeinsamen Erklärung wird als gemeinsamer Ausgangspunkt das Wort Gottes in der Heiligen Schrift, und als erstes Zitat das verheißende Wort in Joh 3,16 (8) genommen. ${ }^{7}$ Alle anderen Schriftbelege folgen dieser Spur, indem hervorgehoben wird, dass die Gläubigen „die Verheißung Gottes immer wieder hören“ müssen, weil sie ständig von Mächten und Begierden angefochten werden (12). ${ }^{8}$ Zwar wird der Zuspruch in der Darstellung des lutherischen Verständnisses von dem Zusammenhang zwischen Sündenvergebung und Erneuerung noch stärker hervorgehoben (23), ${ }^{9}$ aber gemeinsam wird bekannt, dass der „Mensch [...] im rechtfertigenden Glauben auf Gottes gnädige Verheißung, im dem die Hoffnung auf Gott und die Liebe $\mathrm{zu}$ ihm eingeschlossen sind“, vertraut (25). ${ }^{10} \mathrm{Ganz}$

5 So Theodor Dieter in: Der lutherisch/römisch-katholische Dialog und die Gemeinsame Erklärung zur Rechtfertigungslehre (erscheint demnächst). S. a. ebd. „Die Gemeinsame Erklärung hingegen ist eine gemeinsame verbindliche Lehräußerung des Lutherischen Weltbunds im Auftrag seiner Mitgliedskirchen und des Vatikans“.

6 Ich zitiere nach der Endfassung der Erklärung, nachgedruckt in Friedrich Hauschildt/Udo Hahn/Andreas Siemens (Hg.), Die gemeinsame Erklärung zur Rechtfertigungslehre. Dokumentation des Entstehungs- und Rezeptionsprozesses, Göttingen 2009, 273-85.

7 Hauschildt/Hahn/Siemens, Die gemeinsame Erklärung, 275. Im Vergleich zum ersten Entwurf ist das gemeinsame Hören in der Endfassung hervorgehoben. Vgl. a.a.O., 33f. (8).

8 Hauschildt/Hahn/Siemens, Die gemeinsame Erklärung, 277. Vgl. a.a.O., 34 (11). Die Wendung „in Sünde fallen“ ist im Vergleich zur Erstfassung hinzugefügt.

9 Hauschildt/Hahn/Siemens, Die gemeinsame Erklärung, 279. Die Hervorhebung des Zuspruches ist im Vergleich zur Erstfassung hinzugefügt.

10 Hauschildt/Hahn/Siemens, Die gemeinsame Erklärung, 280. Das enge Verhältnis zwischen gnädiger Verheißung und vertrauendem Glauben war hier schon im Erstentwurf zentral. Vgl. a.a.O., 37 (27). 
ähnlich heißt es in Punkt 34: „Wir bekennen gemeinsam, dass die Gläubigen sich auf die Barmherzigkeit und die Verheißungen Gottes verlassen können. "11 Auch hier wird die besondere Weise, in der dieser Aspekt bei den Reformatoren betont wurde, hervorgehoben (35), ${ }^{12}$ aber auch „Katholiken können das Anliegen der Reformatoren teilen, den Glauben auf die objektive Wirklichkeit der Verheißung Christi zu gründen“ und „allein auf Christi Verheißungswort zu vertrauen“ (36). ${ }^{13}$ Damit ist das entscheidend Gemeinsame lokalisiert. Der Text sagt zwar nicht mehr, „Glaube ist Heilsgewissheit“ in der Darstellung der katholischen Lehre wie in der Erstfassung, ${ }^{14}$ aber generell ist die Betonung auf die zentrale Stelle des Gewissheit schaffenden Verheißungswortes von beide Seiten ins Zentrum gerückt.

Der kurze Überblick über den expliziten Gebrauch der Verheißungsterminologie zeigt deutlich, dass das ganze Dokument unter einer verheißungstheologischen Perspektive steht. Der in den Fußnoten durchgeführte Vergleich mit dem Erstentwurf zeigt, dass die verheißungstheologische Perspektive seit Beginn der Arbeit richtungsgebend war. Damit wird das für diese Argumentation Entscheidende gesagt. Die Selbstwahrnehmung der Gesprächsteilnehmer hat sich in diesem Punkt stark aufeinander zubewegt. Wie weit dieses Entscheidende reicht, ist noch zu prüfen. Dass sich aber das Verständnis des Gemeinsamen an diesem Punkt merkbar geändert hat, zeigt ein Vergleich mit der Diskussion über eine gemeinsame Anerkennung der Confessio Augustana zum 450-jährigen Jubiläum von 1980.

Schauen wir z.B. in die von Harding Meyer und Heinz Schütter herausgegebene Dokumentation einer gemeinsamen Untersuchung lutherischer und katholischer Theologen zum Status des Augsburger Bekenntnisses in Kommentarform, ${ }^{15}$ lässt sich leicht feststellen, dass der verheißungstheologische Zugang eine weit geringere Rolle spielt. Im Kapitel „Rechtfertigung - Glaube - Werke“ von Gerhard Müller und Vinzenz Pfnür sucht man auch dort, wo es zu erwarten wäre, vergeblich nach einem Hinweis auf die göttliche promissio. ${ }^{16}$ In dem von Erwin Iserloh und Vilmos Vajta verfassten sakramentstheologischen Beitrag taucht sie

11 Hauschildt/Hahn/Siemens, Die gemeinsame Erklärung, 283. So schon der Erstentwurf. Vgl. a.a.O. 38 (36).

12 Hauschildt/Hahn/Siemens, Die gemeinsame Erklärung, 283. Die Formulierung ist hier präziser geworden. Vgl. a.a.O., 38 (37): „So ist er seines Heils gewiss, wenn auch niemals sicher.“ 13 Hauschildt/Hahn/Siemens, Die gemeinsame Erklärung, 283. Ebenfalls so im Erstentwurf. Vgl. a.a.O. 38f. (38). Auch Theodor Dieter hebt diesen Aspekt besonders hervor in: Der lutherisch/ römisch-katholische Dialog und die Gemeinsame Erklärung (erscheint demnächst).

14 Vgl. Hauschildt/Hahn/Siemens, Die gemeinsame Erklärung, 284 (39), mit a.a.O., 39 (38).

15 Meyer/Schütte, Bekenntnis des einen Glaubens.

16 Gerhard Müller/Vinzenz Pfnür, Rechtfertigung - Glaube - Werke, in: Meyer/Schütte, Bekenntnis des einen Glaubens, 106-139. 
im Zusammenhang mit der Taufe auf ${ }^{17}$ - anders wäre es aber auch kaum möglich. Ebenfalls in sakramentstheologischer Perspektive wird auf die Betonung der Neuentdeckung des Verheißungswortes im Zusammenhang mit der Buße in dem Beitrag Holsten Fagerbergs und Hans Jorissens hingewiesen. ${ }^{18}$

Wenn das Promissionale im reformatorischen Wortverständnis vernachlässigt wird, wird auch das zentrale kommunikative Moment der Rechtfertigungslehre, vor allem in der Relation zwischen Wort und Glaube, übersprungen. Für ein tieferes Verständnis der Sache ist dieser Mangel ein klares Hindernis. Ausnahmsweise hebt Walter Kasper in seinem Beitrag über Bekenntnis und Bekenntnisgemeinschaft in katholischer Sicht zwar die enge Verbindung zwischen Lehre und Vertrauensglauben in Zusammenhang mit der reformatorischen Position hervor, sieht aber in der nachreformatorischen Theologie eine deutliche Tendenz, die existentielle Bedeutsamkeit auf Kosten des „objektiven“ Gehalts zu betonen. ${ }^{19}$ In seiner Darstellung der katholischen Position wird dieses Thema aber im Zusammenhang mit der Frage behandelt, wo die wahre Kirche sei, d.h. als Unterstützung einer römischen Ekklesiologie, und nicht hinsichtlich der Offenbarung und der Zusage der göttlichen Gnade. ${ }^{20}$

Diese kurze Übersicht, die auch durch andere bedeutende Beiträge zur damaligen Diskussion bestätigt werden kann, ${ }^{21}$ zeigt, dass in repräsentativen Texte der 1980er-Diskussion das Wort der promissio eher am Rande im Vergleich mit sakramentstheologischen Topoi oder im Fall Walter Kaspers eher im Zusammenhang mit der katholischen Ekklesiologie entfaltet wird. Im Hinblick auf den Kern, die Rechtfertigungslehre, ist sie nicht zu finden. Dieser Unterschied zwischen 1980 und 1999 ist beachtenswert und in sich selbst verheißungsvoll. Die Entwicklung von sowohl katholischer als auch lutherischer Seite schafft eine bessere Grundlage, um das Gemeinsame in der Confessio Augustana zu finden. Für die katholische Seite ist $\mathrm{u}$. a. hervorzuheben, dass sich innerhalb der katho-

17 Erwin Iserloh/Vilmos Vajta, Die Sakramente: Taufe und Abendmahl, in: Meyer/Schütte, Bekenntnis des einen Glaubens, 198-217, $202 \mathrm{f}$.

18 Holsten Fagerberg/Hans Jorissen, Buße und Beichte, in: Meyer/Schütte, Bekenntnis des einen Glaubens, 228-55, 232f., 236, 238.

19 Walter Kasper, Bekenntnis und Bekenntnisgemeinschaft in katholischer Sicht, in: Meyer/ Schütte, Bekenntnis des einen Glaubens, 23-47, 30.

20 Kasper, Bekenntnis und Bekenntnisgemeinschaft, 41: „Die wahre Kirche ist aufgrund der Verheißung des Herrn eine schon jetzt gegenwärtige Größe“. Damit wird aber hervorgehoben, dass die Verheißung im Dienst der Kirche steht, und nicht umgekehrt.

21 Z.B. spielt die promissio keine Rolle bei W. Pannenberg in seinem Aufsatz: Die Augsburgischen Konfession als katholisches Bekenntnis und Grundlage für die Einheit der Kirche, in: Meyer/Schütte/Mund, Katholische Anerkennung, 9, 17-34. 
lischen Kirche ein differenzierterer Blick auf Luther entwickelt hat. ${ }^{22}$ Aber schon in den Arbeiten der Untersuchung Lehrverurteilungen - kirchentrennend wird die Objektivität des Wortes im Zusammenhang mit der Frage nach der Heilsgewissheit hervorgehoben und in dieser Weise auf die spätere Zentralrolle in der Gemeinsamen Erklärung hingedeutet:

Von hier aus zeigt sich der wahre Sinn der reformatorischen Lehre von der Heilsgewissheit: Weil wir von dem Anspruch des göttlichen Gesetzes, das unsere Werke fordert, von uns aus „subjektiv“ nie bestehen können, soll sich der Glaube auf das „Objektivste“ verlassen, was es für die Kirche gibt: auf das Wort Gottes, wie auch immer es um den Menschen, der sich darauf verlässt, bestellt sein mag. ${ }^{23}$

Um die Confessio Augustana als gemeinsames christliches Bekenntnis anzuerkennen, ist es nötig, das Bekenntnis im Lichte seiner zusammenbindenden Grundorientierung zu verstehen. Hier bietet die Gemeinsame Erklärung sich selbst als Interpretationsschlüssel an, weil sie eben das Element hervorhebt, das nicht nur gemeinsames Herzstück bei Luther und Melanchthon war, sondern, wie im Folgenden gezeigt wird, auch richtunggebend für die Interpretation der Confessio Augustana ist.

\section{Das Wort der Verheißung in der Confessio Augustana}

In der CA 1 wird die gemeinsame Basis des Glaubens in dem dreieinigen Gott selbst festgelegt. Nur wenn es ein Gott ist, kann er in seinen drei ewigen Personen „von unermesslicher Macht, Weisheit und Güte“ in sowohl seinem Schöpfungsals auch in seinem Erhaltungswerk geglaubt werden. ${ }^{24}$ Den trinitarischen Charakter von Luthers Theologie haben Karl Lehmann und Horst Georg Pöhlmann

22 Vgl. Wolfgang Thönissen: Martin Luther in Roman Catholics German-Language Theologies, in: Oxford Encyclopedia on Martin Luther, hg.v. Derek R. Nelson/Paul Hinlicky, Oxford 2017. Schon Walter Kasper konnte auf Grund der Arbeiten von V. Pfnür, W. Pannenberg und H. Jorissen in dem Band Katholische Anerkennung des Augsburgischen Bekenntnisses? Ein Vorstoß zur Einheit zwischen katholischer und lutherischer Kirche, konkludieren, dass „die Augustana zumindest im Licht des Selbstverständnisses heutiger katholischer Theologie nach dem II. Vatikanischen Konzil grundsätzlich katholisch interpretiert und insofern auch katholisch rezipiert werden kann“, a.a.O., 151.

23 Karl Lehmann/Wolfhart Pannenberg/Theodor Schneider (Hg.), Lehrverurteilungen - kirchentrennend?, Freiburg i.B. 1986, 60. S. a. die folgenden Seiten, sowie $56 \mathrm{f}$.

24 BSLK, 50,3-15. 
1980 ganz richtig hervorgehoben, als sie die CA 1, 3 und 16 kommentierten. ${ }^{25}$ Sie fassten nach einer Lektüre der Confutatio zusammen, dass es eine tiefe Übereinstimmung in den fundamentalen Artikeln des christlichen Glaubens gibt, einschließlich der Artikel über die Trinität. ${ }^{26}$ Gleichzeitig machten Lehmann und Pöhlmann darauf aufmerksam, dass trinitarische Theologie in der protestantischen Theologie des 19. und 20. Jahrhunderts eher marginalisiert worden sei. Seit dem Ende des 20. Jahrhunderts würden protestantische Theologen dann jedoch wieder stärker die zentrale Bedeutung der Trinitätslehre für die Reformation betonen. Das ist auch in unserem Zusammenhang von besonderer Wichtigkeit: Je mehr konsensfähige Inhalte in der Confessio Augustana erkannt werden, desto größer wird auch die Basis für eine Verständigung. ${ }^{27}$

Die Erbsünde wird in CA 2 als Mangel an Furcht und Vertrauen gegenüber Gott definiert (sine metu Dei, sine fiducia erga deum). ${ }^{28}$ Das Bekenntnis zum Sohn Gottes in CA 3 entfaltet den verheißenen Inhalt des Evangeliums, der mit dem Glauben als Gewissheit von der Wirklichkeit der Gnade (CA 4) empfangen wird. Notger Slenczka misst CA 7 von der Kirche unter den ersten dreizehn Artikeln eine zentrale Bedeutung zu - zwischen den vorigen sechs Artikeln vom Wort (CA 1-6) und den folgenden sechs Artikeln vom Sakrament (CA 8-13). Wird die CA so gelesen, kann es auch so verstanden werden, dass damit sichergestellt wird, dass auch in den evangelischen Kirchen das Verheißungswort gehört und empfangen werden kann. ${ }^{29}$

Eine solche Darstellung der Hauptgedanken sowohl des Augsburger Bekenntnisses als auch der Gemeinsamen Erklärung ist in der Hervorhebung der konfessionellen Gemeinsamkeiten sehr nahe an den Positionen, die Luther in den Schmalkaldischen Artikeln als unverzichtbar hervorhebt: Sie zeigt die Übereinstimmung in Gotteslehre und Christologie, und ebenso eine Übereinstimmung in dem Artikel, in dem man von nichts weichen oder nachgeben kann, „es fälle

25 Karl Lehmann/Horst Georg Pöhlmann, Gott, Jesus Christus - Wiederkunft Christi, in: Meyer/ Schütte, Bekenntnis des einen Glaubens, 48-78. S. a. Kasper, Bekenntnis und Bekenntnisgemeinschaft, 28f., der den Ursprung der lutherischen Bekenntnisbildung in Luthers trinitarischem Bekenntnis von 1528 ganz richtig verortet.

26 Lehmann/Pöhlmann, Gott, 66.

27 Wenn das Gemeinsame groß genug ist, wird es ein degressives Argument zu behaupten, dass eine wechselseitige Anerkennung die notwendige Rahmenbedingung sei, wie Walter Kasper es behauptet. Die wechselseitige Anerkennung droht damit das gemeinsame Bekenntnis $\mathrm{zu}$ verdrängen. Vgl. Kasper, Bekenntnis und Bekenntnisgemeinschaft, 46.

28 BSLK, 53, 5-8.

29 Vgl. N. Slenczkas Beitrag in diesem Band. 
Himmel und Erden“, d.h. „das Ampt und Werk Jesu Christi oder unser Erlösung“. ${ }^{30}$

Wenn Melanchthon in seiner Apologie zu Artikel 4 zusammenfasst, dass auch die Väter nicht durch das Gesetz gerechtfertigt wurden, sondern per promissionem et fidem, ${ }^{31}$ dann hebt er die zentrale, kommunikative und bekannte Struktur des Wortes als den Mittelpunkt der Rechtfertigung hervor, und damit den engen Zusammenhang zwischen dem Gewissheit schaffenden Wort und dem vertrauensvollen Glauben. Dass dieser Aspekt im Hinblick auf die Interpretation des Bekenntnisses 1980 nicht die Beachtung bekam, die er verdient hatte, wird nochmals ganz deutlich, wenn wir das Kapitel von Müller und Pfnür lesen: Obwohl sie genau auf diese Passage der Apologie Bezug nehmen, indem sie zeigen, wie die Formulierung justificatio per gratiam für die Reformatoren nicht treffend genug war, gehen sie auf die promissio nicht ein. ${ }^{32}$

Um die Bedeutung des Verheißungswortes für die Confessio Augustana in ihrer ganzen Breite und Tiefe zu verstehen, muss man auch den Kontext von Luthers Verheißungstheologie im Horizont des späten Renaissance-Humanismus berücksichtigen. Dieser Rückblick erhellt zugleich den Blick auf die Gegenwart.

\subsection{Das Augsburger Bekenntnis und die Herausforderungen des späten Renaissance-Humanismus}

Wenn wir unsere Gegenwart mit dem Anfang des 16. Jahrhunderts vergleichen, finden wir überraschend viele Gemeinsamkeiten. Nicht nur die revolutionäre Erweiterung der medialen Möglichkeiten verbindet unsere digitale Gegenwart mit der blühenden Publizistik aus den neuen Druckerpressen des 16. Jahrhunderts. Die Beschleunigung der Informationsströme hat damals wie heute einen grundlegenden Skeptizismus befördert. Wie in der Gegenwart fragten sich die Menschen vor fünfhundert Jahren, was als sicheres Wissen gelten kann. Wenn wir den

30 BSLK, 415,21-22. Wir haben in dieser Hinsicht einen noch breiteren Zugang als denjenigen, den Wenzel Lohff 1980 bemerken konnte. Vgl. Wenzel Lohff, Die Bedeutung der Augsburgischen Konfession für die Luthererische Kirche und ihr Verhältnis zur römisch-katholischen Kirche, in: Meyer/Schütte, Bekenntnis des einen Glaubens, 20: „Lässt man Luthers Polemik beiseite, so müsste es aufgrund dieses Modells möglich sein, die nachreformatorische Lehrentwicklung auch in der römischen Kirche neu, offen und nüchtern zu prüfen, ob sie als Interpretation, als Ausdruck des in der Rechtfertigung bezeugten Heilsgeschehens begriffen werden kann. Damit würde zumindest ein Zugang eröffnet zu dem, was in römisch-katholischer Lehrüberlieferung als ,Fülle` des allgemeinen christlichen Glaubens gemeint wird.“

31 BSLK, 171,47-49.

32 Müller/Pfnür, Rechtfertigung, 118. 
wachsenden Skeptizismus im damaligen Denken in den Blick nehmen, wird klar, dass die Reformation nicht nur als eine Kritik an kirchlichen Missbräuchen zu verstehen ist, sondern auch als eine christliche Antwort auf eine generelle epistemologische Krise. ${ }^{33}$

\subsection{Epistemologischer Skeptizismus und das Suchen nach Gewissheit}

Die europäische Spät-Renaissance war auf verschiedene Weise durch einen verbreiteten Skeptizismus herausgefordert. ${ }^{34}$ Dieser wurde durch nominalistisches Denken, einen wachsenden Handel und vermehrte Kriegsführung befeuert, findet seine Grundlage aber vor allem in einem humanistischen Skeptizismus, dessen Wurzeln in der norditalienischen Renaissance liegen. Diese humanistische Bewegung eine Hauptquelle für Unsicherheit und Skepsis. Humanisten wie Lorenzo Valla nährten in der Tradition des Nominalismus die Zweifel, ob ein sicheres Wissen überhaupt möglich sei. Dabei hatten die italienischen Humanisten zwar nicht die Absicht, Sätze der kirchlichen Lehre in Frage zu stellen, dennoch blieb diese Folge nicht aus. ${ }^{35}$ „As historians or using external criticism of texts, humanists could observe how social mores and customs varied across space and time. “ Das brachte ein generell skeptisches intellektuelles Klima hervor. ${ }^{36}$ Obwohl der Humanismus sehr inspirierend für viele Universitäten nördlich der Alpen war, bot er doch keine Antwort auf die Frage nach gesichertem Wissen. ${ }^{37}$ Das zeigte sich vor allem in der Diskussion zwischen Luther und Erasmus.

Wenn wir die Prämisse akzeptieren, dass die Entwicklung einer praktischen Skepsis einen generellen epistemologischen Skeptizismus hervorbrachte, dann wird klar, dass die Reformation sich nicht nur gegen falsche Darstellungen des Evangeliums wandte. Die neue Theologie ist somit zugleich als Antwort auf eine philosophische Debatte zu verstehen, in der die Möglichkeit zuverlässigen Wis-

33 Auch Victoria Kahn weist auf eine Gemeinsamkeit zwischen Gegenwart und Renaissance hin. Sie sieht aber vor allem die Ähnlichkeiten in dem heutigen Streit zwischen Logikern und Pragmatisten in Richard Rortys Terminologie. Victoria Kahn, Rhetoric, prudence and skepticism in the Renaissance, Ithaca u.a. 1985, 190f. Die mediale Entwicklung seit 1985 macht die Gemeinsamkeiten noch größer.

34 Vgl. William John Wright, Martin Luther's understanding of God's two kingdoms: a response to the challenge of skepticism, Grand Rapids, Mich. 2010 (Texts and studies in Reformation and postReformation thought). Wright baut sein Studium u. a. auf die Arbeit von Victoria Kahn, Rhetoric. 35 Wright, Martin Luther's understanding, $56 \mathrm{f}$.

36 Wright, Martin Luther's understanding, 60 with reference to Victoria Kahn, Rhetoric, 27.

37 Wright, Martin Luther's understanding, 94. 
sens grundlegend bezweifelt wurde. ${ }^{38}$ Daraus folgt auch die Möglichkeit, die Augsburger Konfession nicht nur aus der Perspektive des internen Konfessionsverhältnisses zu sehen, sondern auch in dem Kontext einer generellen kulturellen Krise, die beide Konfessionen gleichermaßen betraf. Damit wird nochmals hervorgehoben, dass konfessionelle Kontroversen nicht nur in ihrer Bilateralität zu sehen sind, sondern auch in Blick auf eine gemeinsame Herausforderung interpretiert werden können. Diese gemeinsame Aufgabe ist in dieser Hinsicht aber eine doppelte. Sie besteht erstens in der Verkündigung des Evangeliums, für die mit der Gemeinsamen Erklärung eine überzeugende Grundlage gefunden wurde. Die zweite Aufgabe besteht darin, für die Welt insgesamt eine gemeinsame, von der Last der Ambivalenz befreiende Antwort auf die Möglichkeit gesicherter Erkenntnis zu formulieren. Beide Perspektiven sind wichtig, um einen vollständigen Blick auf die Verantwortung der Kirchen im Umgang mit der konfessionellen Differenz zu gewinnen.

William Wright sieht die sogenannte Zwei-Reiche-Lehre als Angebot zur Bewältigung der Welt, mit der zugleich eine Antwort auf eine grundlegende epistemologische Anfechtung gegeben wird: mit Luthers Unterscheidung des Gotteswortes mit seiner unzweideutigen Vergewisserung des Wohlwollens Gottes von einer Welt der Zweideutigkeit, in die es hineinspricht. Wenn wir Wrights Verständnis von Luthers „Zwei-Regimenten-Lehre“ als eine Antwort auf den epistemologischen Skeptizismus folgen, ${ }^{39}$ dann ist diese Lehre eng mit Luthers neuem Verständnis des Wortes Gottes als promissio verbunden, das als solches die alleinige Quelle der unzweideutigen Gewissheit ist. Sowohl bei Luther als auch bei Melanchthon finden wir ein Verständnis von der promissio, die sowohl das Verspechen einer heilvollen Zukunft ist als auch Gott als den in seiner Verheißung Wohlwollenden und schon gegenwärtig in der sich als zweideutig erfahrbaren Welt Wirkenden bezeugt. ${ }^{40}$ In dieser Weise ist die Verheißung Gottes weitgehend identisch mit der Offenbarung der Barmherzigkeit Gottes.

38 Wright, Martin Luther's understanding, 95.

39 Diese Antwort auf einen epistemologischen Skeptizismus hängt natürlich auch mit Luthers Versuch zusammen, seine Theologie und Schriftlektüre gegenüber Erasmus' hermeneutischem Skeptizismus zu verteidigen. Zu letzterem vgl. Rochus Leonhardt, Skeptismus und Protestantismus. Der philosophische Ansatz Odo Marquards als Herausforderung an die evangelische Theologie, Tübingen 2003 (HUTh 44), v. a. 145-176.

40 Vgl. hierzu Bo Kristian Holm, Luther, Seneca, and benevolence in both creation and government, in: Pekka Kärkkäinen/Olli-Pekka Vainio (Hg.), Apprehending Love: Theological and Philosophical Inquiries, Helsinki 2019 (SLAG 73), 287-312. 


\subsection{Christliche Theologie und römische Sozialphilosophie}

Um den begrifflichen Kontext der promissio zu verstehen, ist es auch notwendig, den intellektuellen Kontext der Reformation weiter zu klären. Es ist festzuhalten, dass Luther bei seinem Versuch, Gewissheit sowohl theologisch als auch epistemologisch neu zu begründen, auch damit begann, das theologische Vokabularium zu erneuern. Es ist in den letzten Jahren deutlich geworden, dass die römische Sozialphilosophie, vor allem in der Gestalt von Senecas De beneficiis und De clementia und Ciceros De officiis, eine bisher unterschätze Rolle in der Entwicklung der reformatorischen Theologie gespielt hat. Wie Risto Saarinen zeigen konnte, hat die Sprache des beneficiums eine entscheidende Bedeutung für die Entwicklung einer neuen theologischen Terminologie in der Reformation. ${ }^{41}$

Luthers Hervorhebung der Gnade als favor dei in seiner Schrift gegen Latomus, die Melanchthon sofort in seine Loci communes von 1521 übernahm, war von einem Senecanischen Verständnis der Benefizien inspiriert. ${ }^{42}$ Die wahre Gabe oder das wahre beneficium sind nicht erst mit der Gabe oder Wohltat als solcher identifiziert, sondern mit dem Wohlwollen oder dem favor, mit welchem es gegeben wurde. Wenn wir die lutherische Rechtfertigungslehre von dieser Perspektive aus betrachten, wird nicht nur die Rolle der promissio innerhalb der lutherischen Theologie deutlicher, sondern auch der Anteil der Gläubigen in der Rechtfertigung, der von katholischen Diskussionspartnern regelmäßig in der Diskussion betont wird. Damit ist die Begrifflichkeit der Rechtfertigung von Anfang an in eine Sprache der Gabe eingeschrieben, in der die gute Gabe sich immer als solche zeigen muss, indem sie den Geber als uneigennützig und wohlwollend offenbart, und der Empfänger die Gabe immer in dem mit der Gabe gegebenen Vertrauen empfangen muss. Auch das könnte dem interkonfessionellen Dialog zu Gute kommen. In dem Moment, in dem zentrale und allzu vertraute theologische Loci durch „neutrale“ Gabe-Kategorien analysiert werden, wird zugleich die gemeinsame christliche Grundlage und damit auch die christliche Fürsorge „für den gemeine Nutzen“ klar erkennbar (vgl. 1 Kor 12,7). ${ }^{43}$

41 Risto Saarinen, Gunst und Gabe. Melanchthon, Luther und die existentielle Anwendung von Senecas „Über die Wohltaten“, in: Johannes Brosseder/Markus Wriedt (Hg.), Kein Anlass zur Verwerfung. Festschrift für O. H. Pesch, Frankfurt a.M. 2007, 184-197.

42 Diese Inspiration ist nicht notwendigerweise direkt zu erkennen. Das wichtigste ist hier die inhaltlichen Übereinstimmungen mit Seneca, die übrigens ganz verbreitet war in der Spätrenaissance, und deswegen zur gebildeten Hintergrundkultur gehörte. Vgl. hierzu: Holm, Luther. 43 Vgl. hierzu vor allem Risto Saarinen, God and the Gift. An Ecumenical Theology of Giving, Collegeville, Minnesota 2005, und die Arbeiten, die in Zusammenhang des interkonfessionellen DFG-Netzwerkes „Gabe: Beiträge der Theologie zu einem interdisziplinären Forschungsfeld“ 
Wenn man die lutherische Rechtfertigungslehre aus der Perspektive ihrer sozialen Dimensionen betrachtet, wird der Anteil der Gläubigen, ein - wie schon betont - für die katholische Seite wichtiger Gesichtspunkt, klar erkennbar. Die Funktion des Vertrauens in die Intention des Gebers ist entscheidend für jede Gabe-Relation, wenn eine Form der Anerkennung von dem Geber auf Seiten des Empfängers intendiert ist. Ein Mangel an Vertrauen in das Wohlwollen des Gebers macht jede Gabe ambivalent. ${ }^{44}$ Wenn Luther von Anfang an Gott als den absoluten Geber versteht, finden wir auch bei ihm eine Aufmerksamkeit auf die notwendige Ambivalenzüberwindung. ${ }^{45}$ So wird bei ihm ein Mangel an Vertrauen in das Wohlwollen des göttlichen Gebers durch das Gefühl des Zornes Gottes charakterisiert und macht es in seinen Augen unmöglich, Gott vom Teufel zu unterscheiden. Wenn an den gebenden Gott mit Vertrauen geglaubt werden soll, ist ein Punkt der Eindeutigkeit notwendig, der den Geber als guten Geber erscheinen lässt und auf den der Glaube sich deswegen stützen kann. So lässt sich das gemeinsame Verständnis des Glaubens im Lehrverurteilungs-Studium gabentheologisch wiederholen: Hier wurde die mögliche gegenseitige Verurteilung des jeweiligen Glaubensbegriffes aufgehoben, indem als entscheidender Punkt die Unterscheidung zwischen Heilsgewissheit und Heilssicherheit hervorgehoben wurde. Vertrauen ist „Heilsgewissheit“, aber nur wenn es in einem Vertrauen auf etwas Externes verankert ist und nicht im Sinne einer „fleischlichen“ Sicherheit verstanden wird. ${ }^{46}$

In ihrem Versuch zur Überwindung der epistemologischen Krise des Renaissance-Humanismus nahmen die Reformatoren Aspekte sozialphilosophischer Diskurse auf und brachten sie in die Behandlung von Glaube, Gnade und

herauskam: Veronika Hoffmann (Hg.), Die Gabe. Ein „Urwort“ der Theologie?, Frankfurt a.M 2009; Veronika Hoffmann/Ulrike Link-Wieczorek/Christof Mandry (Hg.), Die Gabe: Zum Stand der interdisziplinären Diskussion, Freiburg i.B. 2016 (Scientia et religio 14); Veronika Hoffmann, Skizzen zu einer Theologie der Gabe. Rechtfertigung - Opfer - Eucharistie - Gottes- und Nächstenliebe, Freiburg i.B. 2013. Die ökumenischen Möglichkeiten dieser Perspektive sind noch nicht ausgeschöpft, nicht zuletzt zum Thema Aktivität und Passivität und zum Thema Abendmahlslehre bestehen noch Desiderate. Vgl. hierzu vor allem die Diskussionen in Hoffmann, Skizzen zu einer Theologie der Gabe.

44 Vgl. hierzu Bo Kristian Holm, Justification and Reciprocity. „Purified Gift-Exchange“ in Luther and Milbank, in: Bo Kristian Holm/Peter Widmann (Hg.), Word - Gift - Being, Tübingen 2009 (RPT 37), 87-116.

45 Zur Ambivalenzüberwindung bei Luther s. Bo Kristian Holm, Gabe und Geben bei Luther. Das Verhältnis zwischen Reziprozität und reformatorischer Rechtfertigungslehre, Berlin/New York 2006 (TBT 134), $48-69$.

46 Lehmann/Pannenberg/Schneider, Lehrverurteilungen, 62. Vgl. hierzu auch Veronika Hoffmanns Versuch die Rechtfertigungslehre als gegenseitige Anerkennung unter dem Stichwort „Befreiung zur Wechselseitigkeit“ darzustellen: Hofmann, Skizzen, 326-346. 
Rechtfertigung ein. Konkret war dies der Fokus auf wohlwollendes Geben und damit gebendes Wohlwollen. In diesem Adaptionsprozess fügten die Reformatoren einen äußerst unstoischen Aspekt hinzu, indem sie den starken Zusammenhang zwischen Materialität, also der „Gabe“, und Intentionalität, dem favor, betonten. Das wird deutlich, wenn Luther Christus selbst als die Gabe Gottes verstand. ${ }^{47}$

Weil ein Mangel an Vertrauen sowohl menschliches als auch göttliches Geben ambivalent macht, können göttliche Wohltaten nicht nur von der benevola actio abhängig sein, sondern auch vom Glauben des Empfängers. In Luthers Hervorhebung der Inkarnation und des göttlichen Selbst-Gebens wird die enge Relation zwischen „Materialität“, also dem, was gegeben wird, und Intentionalität, in der der Geber als ein wirklicher Geber und nicht als ein Manipulator erscheint, entscheidend. Die Hervorhebung der Intention des Gebers unterstreicht das starke Beharren auf einer notwendigen Externalität der Heilsmittel, Wort und Sakrament. Beide werden als promissio verstanden und deswegen als unzweideutige Qualifizierung des Gegebenen. Von Melanchthon wird in seinen ersten Loci der rechtfertigende Glaube als non aliud nisi fiducia misericordia divinae promissae in Christo erklärt. ${ }^{48}$ Wenige Seiten später setzt er fort „[...] zweifle nicht im Vertrauen darauf, dass du nun keinen Richter mehr im Himmel hast, sondern einen Vater hast, der dich umsorgt, nicht anders als sich unter Menschen die Eltern um die Kinder sorgen“. 49

Melanchthon hat dieses Verständnis vom Glauben vor allem von Luther und aus Hebr. 11,1 gelernt. ${ }^{50}$ Dieses Zentralelement reformatorischer Theologie wird nun dazu benutzt, die Rechtfertigungslehre mit Blick auf die Schöpfung zu öffnen:

Wer so Kraft des Geistes die Schöpfung der Dinge beurteilt, der nimmt auch die Macht Gottes, des Urhebers solcher großen Dinge wie seiner Güte wahr. Wenn er fühlt, dass er alles gleichwie aus den Händen des Schöpfers empfängt, das Leben, die Nahrung und das Kind.

47 Wie z. B. im gedruckten Galaterkommentar von 1519: „,Gegeben“ hat er jedoch nicht Gold und nicht Silber, aber auch nicht einen Menschen, und nicht alle Engel, nein sich selbst. Größeres als das gibt es nicht und hat er nicht." WA 2, 458,3-5. Übersetzung nach Martin Luther, Kommentar zum Galaterbrief 1519, übers. v. Immanuel Mann, Gütersloh 1990 (Calwer Luther-Ausgabe 10). Hervorhebung im Original gesperrt. Vgl. hierzu Bo Kristian Holm, Gabe und Geben, 74-77.

48 Philipp Melanchthon, Loci Communes 1521. Lateinisch - Deutsch. Übersetzt von Horst Georg Pöhlmann, hg.v. Lutherisches Kirchenamt der VELKD, Gütersloh ²1997, 6,22.

49 Melanchthon, Loci Communes 1521, 6,27. Zu diesem und dem Folgenden, s. Holm, Luther. 50 S. z. B. Instructio pro confessione peccatorum, 1518, WA 1,258, 4; Von den guten Werken, 1520, WA 6,209, 25-27. 
Und das [alles] überlässt er dem Schöpfer, damit er es lenkt, regiert, verwaltet und, was er will, nach seiner Güte schenkt. ${ }^{51}$

Die Identität von dem verheißenden Gott mit dem Bild vom barmherzigen Vater im Himmel, der sich zugleich in Kreuz und Auferstehung Christi offenbart, führt zu einem grundlegenden Perspektivwechsel: Sie ändert nicht nur das Verständnis von Gott, sondern auch von der Welt - und eben in dieser Reihenfolge. Die Reformatoren betonen damit zugleich die gegen den Manichäismus formulierte altkirchliche Auffassung, dass auch die zweideutige Welt Gottes gute Schöpfung sei. Mit dieser Betonung von der Präsenz Gottes in den Strukturen des menschlichen Lebens, mit der auch die Güte in einer gefallenen Welt ihren Platz findet, wurde der Gesellschaft gleichzeitig eine allgemeine Idee von Fürsorge für das gemeinsame Gute gegeben. Die Verantwortung der Obrigkeit wurde nach den Pflichten des fürsorglichen Vaters modelliert. Auch hier scheint die Confessio Augustana ein gemeinsames Anliegen beider Konfessionen zu betonen, was u.a. die Studie von George W. Forell und James F. McCue bestätigt.

\subsection{Die Confessio Augustana in der Mitte des irdischen Lebens}

In dem kurzen, abschließenden Beitrag von George W. Forell und James F. McCue über weltliches Regiment und Beruf in der Confessio Augustana in dem Sammelband von Meyer/Schütte beginnen die Autoren mit der Beobachtung, dass diese Abschnitte ,anderer Art als alle übrigen in diesem Kommentar“ sind. Unter den Gegnern der CA in Augsburg habe es hier keinen Widerspruch gegeben. CA 16 über weltliches Regiment wurde „,von den Verfassern der Confutatio ,billich angenommen' (libenter acceptatur)," ${ }^{25}$ was auch Melanchthon explizit bemerkte. ${ }^{53}$ Es scheint zwei gute Gründe zu geben, auf diesen „geringeren“ Artikel zu fokussieren und ihn mit dem epistemologischen Hintergrund, wie er hier bereits im Zusammenhang mit der Spät-Renaissance ausgeführt wurde, zusammenzubringen.

Der erste Grund liegt darin, dass dieser Artikel zunächst das Verständnis für die Gemeinsamkeiten der Gegner der CA in Augsburg eröffnet. In einem weiteren Schritt werden dann auch die Herausforderungen für eine Verständigung zwi-

51 Melanchthon, Loci communes 1521, 6,64.

52 George W. Forell/James F. McCue, Weltliches Regiment und Beruf in der Confessio Augustana, in: Meyer/Schütte, Bekenntnis des einen Glaubens, 319-337 (319).

53 Forell/McCue, Weltliches Regiment, 319; Vgl. Apol 16,1: sine ulla exeptione (BSLK, 307,34). 
schen Gegnern und Befürwortern der CA identifizierbar. Der Ausgangspunkt der Überlegungen ist, dass die christliche Pflicht gegenüber der gemeinsamen Welt gemäß CA 16 durch ein klar anti-machiavellistisches Verständnis weltlicher Autorität geprägt ist. Hier finden wir antike römische Ideale von wohlwollender Obrigkeit, die durch biblische Familie-Metaphern und Gottesvorstellungen gestärkt und mit dem Aufruf zum verantwortlichen Handeln in der Welt gekoppelt werden. ${ }^{54}$

\section{Monastische Theologie und weltliche Verantwortung}

\subsection{Fröhlicher Wechsel und Ehe-Metapher}

Forscher wie Volker Leppin und Berndt Hamm haben unser Verständnis der Wurzeln von Luthers Theologie in der spätmittelalterlich-monastischen Tradition vertieft. ${ }^{55}$ Es wird dadurch deutlich, wie die Reformation zwei bedeutende Linien in der abendländischen Theologie zusammenführte: die monastische und die humanistische Tradition mit ihrer Weiterführung spätantiker sozialphilosophischer Ideale. Diese zwei Traditionen wurden konstruktiv miteinander verbunden, um eine neue theologische Sprache für den Inhalt des christlichen Glaubens zu entwickeln.

Ich möchte diesen Punkt nur insoweit elaborieren, dass ich zeigen kann, wie die Reformation auch im Hinblick auf ihre Position zur weltlichen Verantwortung des Christenmenschen als eine Fortsetzung dessen gesehen werden kann, was Luther im Kloster gelernt hat. In dieser Perspektive wird diese konstruktive Bearbeitung monastischer Theologie entscheidend für das Verständnis des Menschen sowohl in seinem Verhältnis zu Gott als auch zur Welt.

Von seinem Beichtvater, Johannes von Staupitz, hat Luther das seelsorgerliche Ziel der Theologie gelernt, das später das gemeinsame Herz der Wittenberger Theologie werden sollte. Wahre Lehre und wahre Seelsorge wurden eins. ${ }^{56} \mathrm{Im}$

54 Vgl. Holm, Luther.

55 S. Volker Leppin, Die fremde Reformation: Luthers mystische Wurzeln, München 2017.

56 Leppin, Die fremde Reformation, 14, 34-54. Für Melanchthons seelsorgerliche Theologie s. z. B. Timothy J. Wengert, Philip Melanchthon's Annotationes in Johannem in Relation to its Predecessors and Contemporaries, Genf 1987 (Travaux d'Humanisme et Renaissance 220), 154: „The consequences [of stressing certitude] for Melanchton's exegesis are clear. He interprets the promises of Jesus in John's Gospel by investigating their effect on the uncertain conscience of a 
Kloster lehrte Staupitz Luther, sein Heil in dem Gekreuzigten zu sehen. Als Luther die promissio bei der Betrachtung des Kreuzes zu begreifen begann, fand er zugleich das Zentrum seines theologischen Denkens. Er bezog sich fortan wie sein Lehrer Staupitz auf die communicatio idiomatum-Figur, ${ }^{57}$ die auch in der Antiphon zur Vesper und zur Laudes am Weihnachtsoktav in der römischen Liturgie präsent war. ${ }^{58}$ Während nun allerdings der Kirchenvater Augustin mit dieser Figur allein die Betrachtung von Kreuz und Auferstehung verband, liegt in der Antiphon der Schwerpunkt auf der Inkarnation als entscheidendem Akt der Versöhnung. Dieser Punkt gibt der communicatio idiomatum die Funktion als „Achse und Motor“ in Luthers gereifter Theologie. ${ }^{59}$ In dem fröhlichen Wechsel werden Gerechtigkeit und Sünde in einer soteriologischen Parallele zur christologischen Kommunikation der Eigenschaften getauscht. In Luthers Gebrauch kommt der Sprache der Liturgie und Frömmigkeit eine Zentralstelle in der Theologie zu, das gilt vor allem für das Ehe-Bild.

Mit Hilfe der Ehe-Metapher wird der Aspekt der göttlichen Selbsthingabe ein Kardinalpunkt in der Theologie Luthers. Der inkarnierte Christus sowie seine Gegenwart in Brot und Wein werden zum Ausdruck dieser Selbsthingabe, die das göttliche Wohlwollen den sündigen Menschen offenbart. Gleichzeitig wird eben dieses promissionale Selbstgeben die Möglichkeitsbedingung für das menschliche Empfangen dieser Gabe in der Form eines „Zurück-Gebens“ im Glauben. ${ }^{60}$ Das Verständnis von Glauben als fiducia lässt ein spezifisches Verständnis vom

believer beset by ,Anfechtungen‘. In order for the Word to provide certitude, the promise itself must be clear and simple.“

57 S. David C. Steinmetz, Luther and Staupitz. An essay in the intellectual origins of the Protestant Reformation, Durham, N.C. 1980 (Duke monographs in medieval and Renaissance studies 4), 24f., 29, 139.

58 O admirabile commercium!

Creator generis humani,

animatum corpus sumens,

de Virgine nasci dignatus est:

et procedens homo sine semine,

largitus est nobis suam Deitatem.

59 S. hierzu Johann Anselm Steiger, Die communicatio idiomatum as Achse und Motor der Theologie Luthers. Der „fröhliche Wechsel“ als hermeneutischer Schlüssel zu Abendmahlslehre, Anthropologie, Seelsorge, Naturtheologie, Rhetorik und Humor, in: Neue Zeitschrift für systematische Theologie und Religionsphilosophie 38 (1996).

60 Vgl. die einsichtsvolle Studie von Wolfgang Simon, Die Messopfertheologie Martin Luthers. Voraussetzungen, Genese, Gestalt und Rezeption, Tübingen 2003 (Spätmittelalter und Reformation. Neue Reihe 22). 
menschlichen Selbstgeben zu, in dem Vertrauen eine Antwort des ganzen Selbst ist. $^{61}$

Das wechselseitige Selbst-Geben, für welches die Ehe eine Metapher wird, hebt die Relation zwischen der wohlwollenden Intention des göttlichen Gebers und dem Vertrauen des menschlichen Empfängers hervor. Die Bewegung des wechselseitigen Selbst-Gebens ist aus zwei Gründen wichtig. Es wird erstens ein Verständnis menschlicher Passivität im Empfang göttlicher Gnade ermöglicht, das zugleich mit dem Gedanken menschlicher Teilnahme im Ereignis der Rechtfertigung verbunden werden kann, worauf katholische Theologen immer wieder insistieren. Zweitens wird dadurch unser Verständnis der gemeinsamen Verantwortung für das Gute vertieft. Diesem Punkt gilt der folgende Abschnitt, in dem unsere gegenwärtige Verantwortung für die Welt behandelt wird.

\subsection{Bonum commune - politische Wechselseitigkeit im Hohelied}

Luthers Gebrauch des Ehebildes hebt die Symmetrie in der Gott-Mensch-Relation überraschend hervor. ${ }^{62}$ Der Bräutigam gibt sich selbst, und ebenso tut es die Braut. Um den nötigen Unterschied zwischen Gott und Mensch zu sichern, wird bei Luther die Verwendung des Ehebildes mit der Geschichte des Hosea, der eine unwürdige Partnerin zur Frau genommen hatte, kombiniert. In dem wechselseitigen Selbstgeben, das die Rechtfertigung veranlasst, wird der unwürdigen Braut, die nichts zu geben hat, eine neue Würdigkeit und ein neuer Status gegeben, indem sie im Modus des glaubenden Vertrauens sich selbst gibt. Für eine ökumenische Verwendung von Begriffen wie Gabe und Anerkennung ist es wichtig zu wissen, dass Luther dieses Modell der Rechtfertigung in der Lehre von der christlichen Liebe wiederverwendet.

Wie Christus dem Sünder einen neuen Status gibt, ist es beispiels- und bemerkenswerter Weise die Pflicht des Christen in der Ausübung der guten Werke, immer alles, was sein Nächster sagt, in der besten Weise zu deuten (als Auslegung des achten Gebotes), und immer die Lebensbedingungen des Nächsten zu verbessern (als Auslegung des siebten Gebotes).

Dies wird ganz deutlich, wenn wir eine von Luthers politischen Schriften in die Betrachtung einbeziehen. Im Jahr des Augsburger Reichtages hält Luther eine

61 Bo Kristian Holm, Positive Ökonomie als Promissio, in: Hoffmann/Link-Wieczorek/Mandry, Die Gabe, 141-162.

62 Vgl. z. B. Martin Luther, De libertate christiana (1520), StA 2,274,37-275,38. 
fast vergessene Vorlesung über das Hohelied. ${ }^{63}$ In seinem Buch Über Liebe und Herrschaft hat Hans-Martin Gutmann gezeigt, wie Luther in seiner Auslegung das Hohelied vielleicht überraschend für viele als ein politisches Traktat - und nicht anders - liest. Demnach sollte sich die Obrigkeit von Relationen der wechselseitigen Verpflichtungen und im Dienst für das Gemeinwohl auszeichnen. ${ }^{64}$

In Luthers sozialen Vorstellungen des Politischen arbeiten alle Teile der Gesellschaft auf ihrer jeweiligen Ebene für den gemeinen Nutzen zusammen. Luther wendet die mystische Vereinigung der monastischen Theologie zu einer starken sozialen Vorstellung von dem Staat als einem Ganzen, das eng verbunden ist mit der Gegenwart des Schöpfers in seiner Schöpfung, und in dieser Weise ein Grundelement trinitarischen Denkens hervorhebt.

\section{5 „Zum gemeinen Nutzen“ - Vertrauen, Politik und gegenseitige christliche Anerkennung}

Forell und McCue enden ihren Beitrag über weltliches Regiment und Beruf mit einem Hinweis auf die mögliche Orientierungshilfe, die die Confessio Augustana den heutigen Kirchen leisten könnte:

In der CA sehen wir nicht so sehr fertige Antworten auf unsere Fragen als vielmehr wichtige und wertvolle Orientierungspunkte, die, wenn man sie sorgsam beachtet, sich für Lutheraner und Katholiken hilfreich erweisen könnten in ihrem Bemühen, einen Weg zu finden in einer grundlegend gewandelten politischen Welt. ${ }^{65}$

Gewiss hat sich die Welt seit dem Augsburger Bekenntnis geändert. Es gibt aber heute einen dringenden Bedarf für politische und gesellschaftliche Visionen von Vertrauen, Gemeinsamkeit und Einheit zu Gunsten des Gemeinwohls. Wenn Kirchen die Wahrheit in einer Welt voll Falschheit und Misstrauen sagen wollen, sollten sie ernsthaft überlegen jede Möglichkeit zu nutzen, Gemeinsamkeiten zu betonen. Die Confessio Augustana bietet dazu eine Möglichkeit, im Vertrauen darauf, dass auch der Andere auf dem Boden der gemeinsamen Verheißung nichts

63 Martin Luther, Vorlesung über das Hohelied, 1530/31, WA 31 II, 586-769.

64 Hans-Martin Gutmann, Über Liebe und Herrschaft: Luthers Verständnis von Intimität und Autorität im Kontext des Zivilisationsprozesses, Göttingen 1991 (Göttinger theologische Arbeiten 47), 234. S. auch Bo Kristian Holm, Dynamic Tensions in the Social Imaginaries of the Lutheran Reformation, in: Bo Kristian Holm/Nina Javette Koefoed (Hg.), Lutheran Theology and the Shaping of Society. The Danish Monarchy as Example, Göttingen 2018.

65 Forell/McCue, Weltliches Regiment, 332. 
Anderes in dieser Welt sucht als das Gemeinwohl. Dann ist die Frage der katholischen Anerkennung des Augsburger Bekenntnisses auch nicht in erster Linie von bestimmten wechselseitigen Anerkennungsschritten abhängig. ${ }^{66}$ Es zählt vielmehr, ob christliche Kirchen in einer brüchigen und von Skeptizismus belasteten Welt zeigen wollen, wie gegenseitige Anerkennung Konflikte heilen und Vertrauen schaffen kann. Das könnte eine dringende Aufgabe sein: der Welt zeigen, dass die christlichen Kirchen eine gemeinsame Basis in einem eindeutigen göttlichen Versprechen haben, von dem das Augsburger Bekenntnis Zeugnis gibt, und somit auch eine gemeinsame Vision eines Lebens im Dienst des Gemeinwohls.

66 S. z.B. die sonst perspektivreichen Überlegungen zum Begriff wechselseitiger exogener Rezeption, die auch den Rezipienten selbst verändert, in: Ulrich Kühn, Die Frage einer katholischen Anerkennung der Confessios Augustana als Problem ökumenischer Rezeption, in: Hoffmann/ Kühn, Die Confessio Augustana im ökumenischen Gespräch, 21-25. 
